\title{
Capacidad innovadora en la empresa familiar como área de oportunidad hacia el desarrollo de México
}

\section{Innovative capacity in the family business as an area of opportunity toward the development of Mexico}

\author{
Pedro Manuel Rodríguez-Suárez* \\ Beatriz Pico-GonzÁlez** \\ Francisco Javier MÉndeZ-Ramírez ${ }^{* * *}$
}

\begin{abstract}
Family businesses are the business sector $80 \%$ in Mexico, and connote the country's economic dynamism. The aim of this paper derived from a doctoral thesis research is to analyze the relationship between strategic flexibility as a feature role in family businesses, and innovative capacity as an area of opportunity and its impact on development in Mexico, considering the innovative capacity as one of the most valuable intangible capital and entrepreneurialism as a way of life, from conception of the family business to its perpetuity in time.
\end{abstract}

Keywords: family business, strategic flexibility, innovativeness.

\section{Resumen}

Las empresas familiares constituyen del tejido empresarial y connotan el dinamismo económico del país, en México $80 \%$ son de este tipo. El objetivo principal de este artículo, derivado de una investigación de tesis doctoral, consiste en analizar la relación entre la flexibilidad estratégica -como una característica preponderante en las empresas familiares- y la capacidad innovadora como una área de oportunidad y uno de los más valiosos capitales intangibles de impacto en el desarrollo de México, y a la actitud emprendedora como una forma de vida, desde la gestación de la empresa familiar, hasta su perpetuidad en el tiempo.

Palabras clave: empresa familiar, flexibilidad estratégica y capacidad de innovación.

\footnotetext{
* Benemérita Universidad Autónoma de Puebla, México. Correo-e: pedrosuarezbuap@yahoo.com

** Universidad Popular Autónoma del Estado de Puebla, México. Correo-e: beatriz.pico@ upaep.mx

*** Benemérita Universidad Autónoma de Puebla, México. Correo-e: fjmendez@itesm.mx
} 


\section{Introducción}

De acuerdo con estadísticas sobre empresas familiares, en los países occidentales como el Reino Unido 75\% de las empresas son de este tipo, en Alemania y España, 80\%, mientras que en Suiza fluctúa de entre 85 y $90 \%$, finalmente, en Italia 99\% de los negocios son familiares (Poulain, 2006). En México, 90\% de las empresas son consideradas de tipo familiar y equivalen a más de 3.6 millones de empresas, lo cual es un importante impulsor de la economía en este país (INEGI, 2011).

El concepto de empresa familiar, de manera sencilla, implica la relación intrínseca entre dos sistemas -empresa y familia-, pero es más complejo y sistémico, puesto que la empresa familiar desde su gestación, madurez y desarrollo en el tiempo se puede analizar desde distintos puntos de vista que van ligados al desarrollo empresarial y familiar. En este sentido, el objetivo principal de este artículo es presentar la necesidad existente, dentro de las empresas familiares, de resaltar la flexibilidad estratégica como una de sus principales fortalezas y mostrar cómo, combinada con la capacidad innovadora, pueden impactar en el desarrollo económico y social en México.

$\mathrm{Al}$ considerar que en una empresa familiar participan dos o más miembros consanguíneos que ejercen un papel en la vida y funcionamiento de dicha corporación, la naturaleza y medida de la colaboración trae como consecuencia que ésta sea variable: en algunos casos ciertos miembros de la familia trabajan un tiempo parcial, sin que esto impida que adquieran un alto compromiso empresarial, relacionado de manera positiva con la flexibilidad estratégica, que es la capacidad de buscar nuevas oportunidades y responder a las amenazas que se presentan en el entorno competitivo (Zahra, et al., 2008).

Otro factor importante es la capacidad innovadora: los autores Mahmoud, y Mignon (2010) realizan el estudio: "Capacidades de innovación de la empresa familiar duradera”, en donde subrayan cuatro características de las empresas con tendencia a la capacidad de innovación, las definen como las cuatro $\mathbf{C}$; la primera es la continuidad, que se explica como la habilidad de manejar proyectos a largo plazo de alto impacto y confrontar de manera exitosa la sucesión de una generación a otra; la segunda es la colectividad, ésta se define como la destreza de trabajar en conjunto con empleados ajenos a la familia y familiares dentro de la organización de manera alineada. En tercer lugar, la conexión que significa mantener un adecuado sistema de comunicación entre los integrantes de la organización, y por último, el control especifico, definido como la destreza para controlar las decisiones de tipo estratégico y económico de una manera prudente y consensuada. 
La actitud emprendedora, como piedra angular de la flexibilidad estratégica, puede considerarse un factor clave para el desarrollo corporativo de un empresario: sus habilidades, conocimientos, capacidades, experiencia y valores son agentes detonantes de la actitud empresarial que se forja al inicio y durante el desarrollo; asimismo, esta actitud es una conducta de iniciativa para propiciar el constante desarrollo de ideas creativas y generar mejoras en su entorno. La tendencia de mantenerse alerta de los cambios en su medio de acción se relaciona con la habilidad para detectar oportunidades, la propensión para asumir riesgos, así como la tolerancia al fracaso, vista como una cualidad mediante la cual se deduce que siempre existe un método mejor para hacer las cosas y la capacidad de innovación como el conjunto de cualidades individuales y de grupo para realizar con éxito cambios radicales o incrementales en un ámbito determinado.

La actitud emprendedora tiene un alto impacto en la gestación de la empresa, y forma parte de una característica del fundador o primera generación, esta actitud es una de las bases de la flexibilidad estratégica, y asimismo se convierte en capacidad innovadora cuando la empresa enfrenta procesos de cambio para sobrevivir a las exigencias del mercado, en el entendido de que innovar no es precisamente tecnología, sino además nuevas formas de administrar, vender, fabricar y brindar un producto o servicio.

La actitud emprendedora también puede ser un factor importante dentro de la segunda o tercera generación de la empresa, ya que son las que pueden aportar nuevas ideas, y es probable que estas generaciones estén influenciadas por agentes externos como la formación académica, empresarial y cultural, que combinados con la fuerte influencia de educación familiar tienden a esta inercia por el cambio.

La empresa familiar es un complejo sistema que hace que cada empresa sea diferente y tenga procesos empresariales peculiares, existen historias muy diversas en donde no necesariamente la capacidad innovadora fue inducida por los miembros familiares, puede haber sido por algunos de los empleados no familiares quienes apoyados por la familia prosperaron y desarrollaron alguna iniciativa empresarial.

\section{Metodología}

La metodología que se llevó a cabo para la elaboración de este artículo fue explicativa y descriptiva, resultado de investigaciones publicadas empíricas y teóricas, que fueron analizadas y sintetizadas. Los resultados de las mismas presentan una frecuencia en las variables que de una forma simple muestran repetición por parte de los diferentes autores y del cual 
se obtuvieron diferentes resultados que generaron un modelo conceptual así como su discusión. La experiencia en materia de consultoría en empresas familiares sirve de referente importante para contextualizar lo expresado en este documento.

Cabe resaltar que existen investigaciones en América Latina en torno a la empresa familiar, en Brasil se realizó un análisis de la producción de artículos científicos relacionados con la empresa familiar entre 1997 y 2007. Al respecto, se encontraron 83 artículos en total de los cuales los temas más destacados fueron: la sucesión, estrategia, modelos de gestión, profesionalización, cultura de aprendizaje, las representaciones sociales, el cambio, el espíritu empresarial y los sistemas de contabilidad. Asimismo, se observó el predominio de los estudios teóricos y empíricos. Los sectores económicos más estudiados fueron empresas de servicios e industria. En cuanto al tipo de enfoque, el cualitativo fue el más recurrente, utilizando la entrevista y recopilación de datos como principal herramienta. Por último en cuanto a sus fuentes consultadas, la mayoría hace referencia a publicaciones nacionales brasileñas (Paiva et al., 2008).

Por tanto, la revisión de la literatura que se detalla en este documento muestra una trayectoria de trabajos de exploración acerca de autores de empresa familiar y su implicación en las variables que se presentan en éstas, como son: la flexibilidad estratégica y la capacidad innovadora; en cada una se ha argumentado que existe una sólida relación entre estas variables.

\section{La empresa familiar y el desarrollo económico y social de México}

\section{1. ¿Qué es una empresa familiar?}

Se define como aquella empresa que la mayor cantidad de su capital accionario se encuentra en posesión de una familia, así como la presencia de familiares en el cuerpo directivo de la empresa. Esta influencia puede ser tanto de la familia directiva que participa en la gestión de la empresa $\mathrm{o}$ indirectamente se refiere a dirigentes familiares que tienen un poder dentro de la cultura corporativa y la cultura de interacción familiar (Poulain, 2006).

Existen tres ámbitos mediante los cuales se debe entender a la empresa familiar, el primero es la propiedad donde se identifica quién o quiénes son dueños de la empresa por medio de capital accionario, o de otra manera, quienes ostentan ser dueños de los activos más importantes. El segundo tiene que ver con la familia, en este ámbito se identifica quienes conforman la familia y cuántas generaciones existen en ella, así como lo compleja que pueden resultar la mayoría de las veces sus relaciones inter- 
personales, ya sea entre miembros consanguíneos o familiares políticos. Por último, la empresa, en la cual es importante resaltar y analizar los logros o retos que tiene por delante la misma, en algunas ocasiones en este ámbito se ve su desarrollo estancado por el tipo de liderazgo que mantiene el fundador de la empresa y la pugna de la segunda generación intentando hacer el cambio (Tagiuri y Davis, 1982).

Por otro lado, puede presentarse el caso de organizaciones que se encuentran en un estado de crecimiento y el trabajo conjunto de sus integrantes, familiares y no familiares, resulta tener cierta armonía y alineación estratégica con la visión del fundador, no dejando de observar las áreas de oportunidad que se puedan presentar para estas organizaciones. Cada empresa familiar tiene una historia que contar y su complejidad es fascinante en la medida en que se toma como una forma de vida: la filosofía el oficio, trabajo, o el giro de la organización, y se convierte en la parte más importante en la vida de quienes, como familia, están involucrados en ella.

\subsection{La micro, pequeña y mediana empresa en el desarrollo económico y social de México}

En México la importancia de las micros, pequeñas y medianas empresas es vital, la mayor cantidad de empleos que se ofrecen en este país son derivados de éstas. La mayoría se dedican a los servicios no financieros y al comercio, llegando a sumar las primeras 1,361,945, y las segundas, $1,854,197$. No por ello es menos importante la actividad manufacturera que suma 433,618 empresas; entre las actividades empresariales de cada sector se describe de la siguiente manera:

Manufacturas: alimentos, bebidas y tabaco, textiles, prendas de vestir e industria del cuero; madera y sus productos; papel, productos de papel, imprentas y editoriales; sustancias químicas y artículos de plástico o hule; bienes a base de minerales no metálicos; industrias metálicas básicas; productos metálicos, maquinaria y equipo, así como otras industrias manufactureras.

Comercio: materiales de desecho; no alimenticios al por mayor; alimenticios al por mayor; alimenticios al por menor; supermercados; no alimenticios al por menor; tiendas departamentales; automóviles y gasolineras.

Servicios: transacción de bienes raíces; alquiler de bienes muebles; servicios educativos, médicos, de asistencia social y organizaciones; restaurantes y hoteles; servicios culturales, deportivos y recreación; servicios profesionales, técnicos, personales y domésticos; servicios 
de reparación y mantenimiento y servicios relacionados con las actividades económicas (INEGI, 2011).

En la mayoría de los países del mundo, las Pymes constituyen 90\% del total de las empresas. En la Unión Europea y Estados Unidos representan 95\% de las unidades económicas y 75\% de los empleos; en América Latina se tienen estimaciones entre 35\% y 40\% de la generación de empleo de esta región. A nivel nacional en México generan 52\% del PIB (Pavón, 2010).

El impacto social más importante de las Pymes está representado en las fuentes generadas de empleo, mismas que son reportadas por INEGI ${ }^{\mathrm{I}}$. Para el último censo económico (2009) reportaba 3,656,000 empresas en total de las cuales $99.7 \%$ son micros, pequeñas y medianas, así también se puede observar el crecimiento que existe de un censo a otro de las pequeñas y medianas empresas (cuadro 1).

Es de suma importancia que cada vez se integren a la actividad económica nuevas empresas como el caso de las micro, pero el que sobrevivan al primer año de operaciones es aún más relevante ya que ocho de cada 10 empresas cierran antes de este tiempo (Pavón, 2010).

\subsection{Impacto económico y social}

Millones de personas a través de la existencia de las micro, pequeñas y medianas empresas, proporcionan el diario sustento a sus familias y muchas de ellas desarrollan actos emprendedores, un medio cada vez más complicado y necesario en el mundo laboral. El emprendedor inicia un negocio, que en ocasiones puede fracasar o tener éxito, si sucede lo primero seguramente lo llevará a una aventura empresarial, si no probará una y otra vez, ya que al haberse independizado no siempre decide regresar a la cotidianidad del sueldo fijado por otra persona.

En este afán de concebir una actividad que genere ingresos propios, se ve en la necesidad de improvisar desde instalaciones físicas, hasta el mismo proceso productivo o de servicio, involucrando con ello capital humano, familia, esposa e hijos y, en muchos otros de los casos, a hermanos y primos.

Por otro lado, la interrelación que existe entre la micro, pequeña y mediana empresa con la familia está en la gestación de la empresa, es decir, en sus orígenes; puede haber unas empresas más jóvenes que otras, y muchas mueren en el intento. En este tipo de empresas existen lazos

${ }^{1}$ El Instituto Nacional de Estadística, Geografía e Informática en México realiza censos económicos cada cinco años para dar cuenta de la actividad económica de las empresas. 


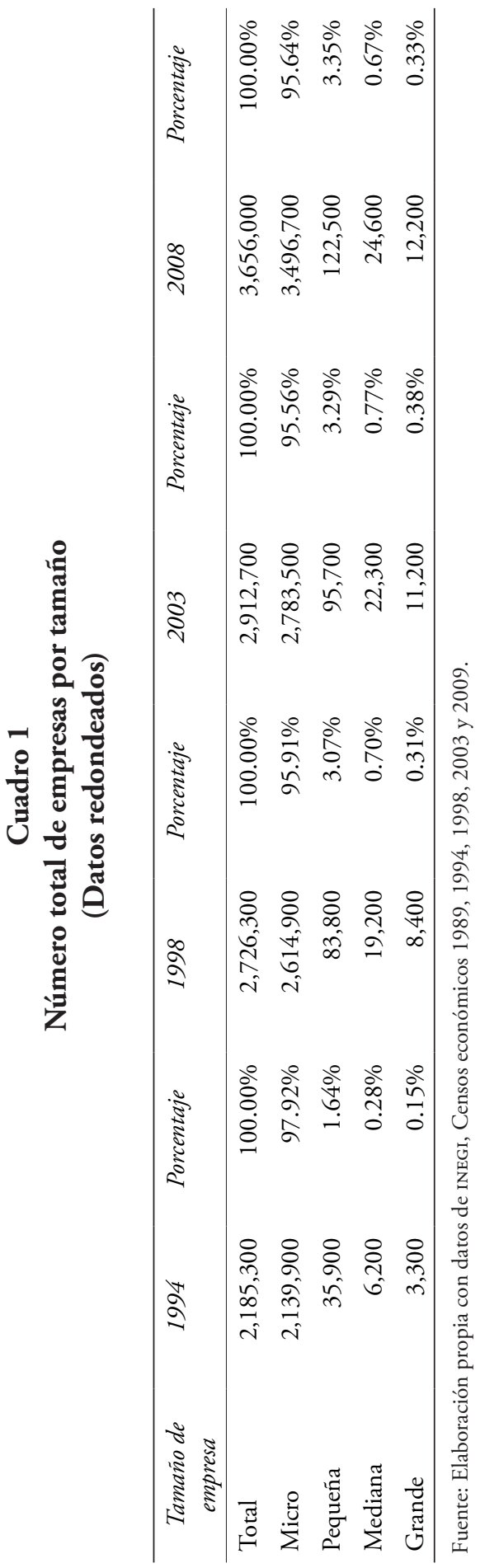


familiares a lo largo y ancho de la organización, en donde el directordueño suele ser el padre, y los hijos u otros parientes se ubican en mandos subordinados.

Con base en lo anterior, la influencia del comportamiento de las empresas familiares impacta en el desarrollo económico de un país a través de la capacidad de crear riqueza, asimismo promueve y mantiene la prosperidad o bienestar económico y social de sus habitantes por medio de la generación de empleo de ahí la importancia de las empresas con un perfil familiar dentro de la economía en México.

\section{Flexibilidad estratégica y capacidad innovadora en las empresas familiares, el binomio adecuado}

Las empresas familiares, como generadoras de un alto impacto en el desarrollo económico de los países son parte importante de la competitividad del mismo; por tal motivo, la flexibilidad estratégica representa un activo muy valioso en entornos competitivos, ésta se define como la habilidad de una organización para adaptarse a cambios del entorno con alto impacto en el desempeño de las organizaciones (Lloréns, et al., 2006). Por lo tanto, la flexibilidad estratégica y la capacidad innovadora, combinadas pueden dar como resultado empresas familiares de largo plazo; tomando en cuenta que la flexibilidad estratégica está directamente relacionada con la ventaja competitiva de la empresa, ya que es la característica estratégica más importante porque define al producto o servicio como único, difícil de imitar, posible de mantener mediante estrategias de innovación netamente superior a la competencia.

La capacidad de innovación es una característica subyacente de las empresas familiares, la cual forma parte de una cultura o filosofía reflejada como una forma de vida para la organización, para que esto exista debe haber condiciones o un ambiente innovador, por ejemplo, anticiparse a las tendencias futuras y reaccionar de manera proactiva al cambio, generar o crear un sentido de pertenencia y compromiso dentro de los colaboradores, integrar equipos multidisciplinarios de trabajo, responder con rapidez a las necesidades del cliente, producir soluciones que creen valor económico reflejadas en productos y servicios, y tener la habilidad de resolver conflictos interpersonales dentro de la organización (Colina, 2008). El innovar en su esencia más simple es tener la capacidad de desplazar los recursos valiosos de la empresa hacia las oportunidades para obtener un mayor valor de mercado.

Así también se ha identificado que muchas empresas familiares son el resultado de un acto emprendedor, es decir, que una persona decidió tener la iniciativa de fundar una empresa que a la postre es una empresa 
familiar, aunque es importante acotar que iniciar una empresa no necesariamente es ser emprendedor sino empresario, y que la capacidad administrativa y el respaldo de la familia modelará el futuro inmediato de la empresa (Lozano y Zatarain, 2011).

\subsection{La flexibilidad estratégica}

La capacidad con la que cuentan las empresas familiares para identificar oportunidades y responder a los cambios puede ser la clave de una ventaja competitiva. Así también aquellas empresas que se caracterizan por ser conservadoras a menudo carecen de capacidad de adaptación. De ahí que las empresas familiares con un alto compromiso empresarial se relacionan de manera positiva con la flexibilidad estratégica, y tienen la capacidad de buscar nuevas oportunidades y responder a las amenazas dentro del entorno competitivo (Zahra, et al., 2008).

Es así como Farías Adán define la flexibilidad estratégica: "el conjunto de capacidades y habilidades de la organización para variar, ajustar e instaurar determinadas prácticas de gestión que se manifiesta en la búsqueda, generación y evaluación de soluciones y en el reajuste de objetivos con rapidez, libertad de acción y susceptibilidad" (Farías, 2008: 3). Por lo tanto, la flexibilidad estratégica está determinada por el nivel de compromiso de la familia hacia la empresa, así como la adopción de prácticas de trabajo orientadas a la administración y diseños organizacionales para atraer talento humano externo. Un estudio realizado en Estados Unidos de Norteamérica por Zahra, et al., (2008) en 248 empresas mostró que la flexibilidad estratégica podría lograrse de varias maneras, donde las empresas familiares se benefician de tener una cultura valorando la participación de los miembros de su familia en su proceso de toma de decisiones y la adopción de una perspectiva responsable en la gestión de sus operaciones.

Las variables que determinaron la elaboración de este estudio son: la cultura familiar de las empresas consideradas desde los roles de la familia, implícitos éstos dentro de la dinámica laboral de la empresa, el compromiso hacia la cultura empresarial reflejado en factores, pensamientos y creencias alrededor de motivaciones hacia recompensas y sistemas de control aunado a la flexibilidad estratégica valorada como la caraterística distintiva por la que una empresa está dispuesta a cambiar su estrategia en respuesta a las oportunidades, amenazas y cambios del exterior.

Por otra parte, Farías Adán (2008) considera las cinco dimensiones de la flexibilidad estratégica, las cuales define como: la dimensión temporal, el tiempo que le toma a una organización ajustar o reajustar nuevas condiciones desde un punto de vista activo o reactivo. Muchas organizaciones 
Pymes, y sobre todo las familiares, tienen poco tiempo para tomar una decisión de cambio, por la rapidez de comunicación que existe, o en su defecto no tomarla y, en algunas ocasiones, dejar pasar una oportunidad.

Asimismo, en las empresas familiares la influencia que existe del liderazgo del fundador en los empleados familiares y no familiares es predominante, consiste en la capacidad de las personas para cambiar las percepciones, habilidades y competencias de acuerdo con el momento contextual de la organización, o también es una caraterística que puede ser desarrollada en la segunda generación como una clara combinación de la visión estratégica del fundador y las nuevas ideas y formas de llevar a cabo las acciones; la dimensión operacional está íntimamente ligada al grado de capacidad y de ajuste según el contexto de operaciones de la empresa mutuamente relacionadas, por lo que en las empresas familiares existe una clara tendencia para enfocar al cliente y esto se ve reflejado en la forma de brindarle un producto o servicio que cumpla cabalmente con sus expectativas; la dimensión contextual va dirigida hacia aquellas acciones que puede recibir o experimentar una determinada organización hacia el interior y el ajustarse a ellas con el fin de adquirir un aprendizaje organizacional, las organizaciones que aprenden de sus errores y sobre todo lo documentan de tal forma que puedan capitalizarlo como un aprendizaje formando parte de su capital intelectual; finalmente, la dimensión económica define los costos económicos asociados para enfrentar los cambios, una cultura financiera enfocada a presupuestar partidas económicas destinadas al desarrollo de la innovación es una parte fundamental para las organizaciones actualmente.

\subsection{Capacidad de innovación}

La capacidad de innovación es una característica distintiva de las empresas familiares ya que la concepción inicial de muchas de ellas es el resultado de la concreción de una idea plasmada en un producto, servicio o idea de negocio, razón por la cual Javier Quintana (2007: 103-130), del Instituto de la Empresa Familiar en España, hace las siguientes aseveraciones con base en la innovación:

Primero: No invertir en innovación es una opción válida, pero, al contrario de lo que suele pensarse, es la vía más arriesgada. En la sociedad del conocimiento mantener una estrategia estática implica darle ventajas a la competencia.

Segundo: Innovar por innovar no sirve. La empresa familiar que desee tener éxito a la hora de trabajar en esta actividad debe planificar y 
realizar un ejercicio en el que alcanzar la solución correcta no es sencillo.

De esta manera se puede observar que la capacidad de innovación depende de las condiciones con las que cuente la empresa familiar para afrontar este tema. Esta capacidad es un reflejo de las definiciones siguientes generadas por los autores franceses Mahmoud y Mignon (2010):

1. Una visión clara de largo plazo combinada con una cohesión familiar en el sentido de desarrollar una diferenciación en el mercado y generar valor a través de las generaciones.

2. Los valores están a menudo basados en una sólida ética personal de liderazgo, marcada por la dedicación, responsabilidad, capacidad de trabajo y el significado del éxito.

3. Interrelaciones empresa/familia fuertes y sólidas. La empresa familiar es vista como un sistema compuesto de: a) un subsistema consistente de la historia familiar, tradiciones, ciclo de vida familiar, b) un subsistema, incluidas las estrategias de empresa y estructuras establecidas para crear valor y c) un subsistema integrado por personas, miembros de la familia caracterizado por sus intereses, sus habilidades y su nivel de participación en el control de procesos y gestión.

4. Administración. La implicación emocional de la familia en la dirección. Esta gestión se caracteriza por la identificación con la organización y sus objetivos, el compromiso personal del líder para el éxito de su empresa, el sacrificio personal, la motivación para actuar por el bien y para el interés colectivo, accionistas y empleados.

5. Una asignación de recursos prudente y control de riesgos. La toma de decisiones de largo plazo, la participación y la lealtad del personal y cierta paciencia en términos de retorno sobre la inversión; son todos los componentes de familiares que fortalecen su reputación y evitan tener riesgos. Además, si éstos van acompañados de estrategias financieras prudentes y una correcta evaluación de proyectos.

6. Una estabilidad de las relaciones externas. Relaciones con los clientes, proveedores, bancos y en general con socios estratégicos. Estas relaciones se ven facilitadas por la continuidad de los directivos, lo que facilita la construcción de la confianza y pone a prueba la duración de los compromisos contraídos.

La flexibilidad estratégica y la capacidad de innovación son agentes mutuamente complementarios entre sí, ya que el primero es la caracterís- 
tica distintiva de aquellas organizaciones que por su estructura empresarial están dotadas de una cultura que les permite anticiparse a los cambios y estar en vigilancia estratégica con respecto a las oportunidades que suceden en su entorno. Por otro lado, la capacidad de innovación tiene como base el talento humano de la organización, que incluye la capacidad de estar en constante búsqueda de procesos de mejora, conscientes del valor de su capital intelectual en términos de la habilidad de perpetuar y acumular este conocimiento para las futuras generaciones, así como estar abiertos al exterior y tomarlos para la capitalización del conocimiento como una estrategia financiera de inversión a largo plazo y, por último, generar un ambiente de total libertad, donde se expongan las ideas sin ningún tipo de restricción.

\section{Conclusiones}

El capital más importante que tiene un país es su gente ya que permite observar un mosaico de distintas posibilidades y puntos de vista; asimismo, la empresa familiar forma parte importante de la vida económica de muchos países, y son estas empresas que por medio de las familias hacen historia a través de sus productos y servicios, que en algunos casos son tradiciones obligadas para conocer, comprar y experimentar en diversas poblaciones de México y el mundo.

Por lo tanto, la flexibilidad estratégica y capacidad innovadora desempeñan un papel indispensable en las empresas familiares para propiciar su continuidad y desarrollo, así también existen otras empresas que no las consideran características preponderantes, sin embargo, puede representar una área de oportunidad a desarrollar, siempre y cuando sean reconocidas las condiciones por la empresa y estén dispuestas a desafiarlas. La cultura emprendedora, como pilar substancial de la flexibilidad estratégica, resulta un factor relevante, inspirador y brinda fortaleza para enfrentar atractivos desafíos en la historia y evolución de la empresa. Lo más importante es identificar las variables antes descritas dentro de la empresa familiar, haciendo un acto de reflexión en éstas y colaborando para que se identifique y se potencialicen para su desarrollo y continuidad, no existe nada más importante para un país que fortalecer su tejido social por medio del trabajo y oportunidades de desarrollo, y qué mejor que sea a través de la empresa familiar.

Es así como las empresas familiares se pueden analizar desde diferentes perspectivas, en concreto, en el caso mexicano resulta conveniente estudiar a la empresa familiar desde un punto de vista cualitativo y cuantitativo, como es el caso del análisis decisional y su relación con las estrategias internas y el impacto generado en las estrategias externas; otro 
aspecto que no ha sido investigado a profundidad es el desarrollo de la sustentabilidad y su relación con la productividad.

Una de las aportaciones principales en el desarrollo de esta investigación documental por parte de los autores fue la realización del análisis cualitativo entre las variables flexibilidad estratégica y la capacidad para innovar la empresa familiar, considerando que el uso constante de la primera produce la diferencia que la hace única en relación con la competencia, generando un impacto en su desarrollo.

\section{Bibliografía}

Colina-Arenas, Belinda (2008), "Capacidades societales de innovación. Su desarrollo en empresas de producción social en el contexto del socialismo del siglo XXI en Venezuela", Revista de economía pública, social y cooperativa, núm. 60, Cieriec-España, Maracaibo. pp. 121-153.

Farías-Adán, Jorge Antonio (2008), "La flexibilidad estratégica", Técnica Administrativa, núm. 35, vol. 7, julio, Técnica Administrativa e-journal, Buenos Aires, URL http://www.cyta.com.ar/ta0703/ v7n3a3.htm ISSN 1666-1680, 7 de julio de 2013.

INEGI (Instituto Nacional de Estadística, Geografía e Informática) (1989), Micro, pequeña, mediana y gran empresa. Estratificación de los establecimientos. Censos Económicos, INEGI, Aguascalientes

INEGI (Instituto Nacional de Estadística, Geografía e Informática) (1994), Micro, pequeña, mediana y gran empresa. Estratificación de los establecimientos. Censos Económicos, INEGI, Aguascalientes

INEGI (Instituto Nacional de Estadística, Geografía e Informática) (1998), Micro, pequeña, mediana y gran empresa. Estratificación de los establecimientos. Censos Económicos, INEGI, Aguascalientes

INEGI (Instituto Nacional de Estadística, Geografía e Informática) (2003), Micro, pequeña, mediana y gran empresa. Estratificación de los establecimientos. Censos Económicos, INEGI, Aguascalientes

INEGI (Instituto Nacional de Estadística, Geografía e Informática) (2009), Micro, pequeña, mediana y gran empresa. Estratificación de los establecimientos. Censos Económicos, INEGI, Aguascalientes 
INEGI (Instituto Nacional de Estadística, Geografía e Informática) (2011), Micro, pequeña, mediana y gran empresa. Estratificación de los establecimientos. Censos económicos 2009, INEGI, Aguascalientes.

Lloréns-Montes, Javier, Antonio Verdú-Jover y Víctor García-Morales (2006), "Flexibilidad estrátegica en entornos hipercompetitivos: una visión basada en los recursos financieros de la empresa", Revista Española de Financiación y Contabilidad, xxxv (129), Asociación española de contabilidad y administración de empresas, Madrid, pp. 387-409.

Lozano-Carrillo, Óscar, Niebla Zatarain y Juan Cayetano (2011), "El emprendedor de la empresa familiar mexicana", Gestión y estrategia, núm. 40, Universidad Autónoma Metropolitana Azcapotzalco, México, pp. 65-72.

Mahmoud-Jouini, Bloch y Sophie Mignon (2010), "Capacités d'innovation des entreprises familiales pérennes”, Revue Française de Gestion, núm. 200, Cedex, France, pp.111-126.

Paiva, Kely César Martins de, Michelle Cristina de Souza Mendes de Oliveira y Marlene Catarina de Oliveira Lopes Melo (2008), "Produçao cientifica brasileira sobre empresa familiar -um metaestudo de artigos publicados em anais de eventos da anpad no periodo de 1997-2007-", revista de Administraçao Mackenzie, núm. 9, Universidade Presbiteriana Mackenzie, São Paulo, pp. 148-173.

Pavón, Lilianne (2010), Financiamiento a las microempresas y Pymes de México (2000-2009), Comisión Económica para América Latina y el Caribe, Naciones Unidas, Santiago de Chile, p. 69.

Poulain-Rehm, Thierry (2006), “Qu'est-ce qu'une entreprise familiale? Réflexions théoriques et prescriptions empiriques", La Revue des Sciences de Gestion, Direction et Gestion, núm 25, ProQuest European Business, Bordeaux, pp. 77-87.

Quintana, Javier (2007), "La innovación en las empresas familiares", CLM-Economía, núm.7, Revista Económica de Castilla de la Mancha, Toledo, pp. 103-130. 
Tagiuri, Renato y John Davis (1982), "Bivalent attributes of the family firm”, Family Business Review, núm. 4, Harvard Business School, SAGE Publications, Thousand Oaks, California, pp. 199-208.

Zahra-Shaker, James Hayton, Donald Neubaum y Justin Craig (2008), "Culture of family commitment and strategic flexibility: The moderating effect of stewardship", 32(6), Entrepreneurship theory and practice, Baylor University, Waco, pp. 1035-1054.

Recibido: 24 de febrero de 2011. Reenviado: 4 de diciembre de 2012. Reenviado: 4 de diciembre de 2012. Aceptado: 24 de enero 2013.

Pedro Manuel Rodríguez-Suárez. Doctor en estudios de integración europea por la Universidad de Varsovia, Polonia. Egresado de la licenciatura en relaciones internacionales por la Universidad Iberoamericana, ciudad de México; diplomado en estudios europeos por el itam. Fue coordinador de la licenciatura en relaciones internacionales en la Universidad del Mar y en la Universidad Iberoamericana. Actualmente es profesor-investigador de tiempo completo de la Universidad Benemérita Autónoma de Puebla. Miembro del Sistema Nacional de Investigadores nivel 1. Sus líneas de investigación son: cooperación internacional, regionalismo y desarrollo regional. Coordinador y autor del libro: América Latina: regionalización, cooperación internacional y problemas medioambientales, Benemérita Universidad Autónoma de Puebla-Universidad Autónoma de San Luis Potosí, México (2012); autor del libro Hacia una nueva Europa la integración de los paises de Europa Central y Oriental en la Unión Europea, Fondo de Cultura Económica (2006); coautor del libro Las relaciones de México y la Unión Europea: retos y perspectivas, El Colegio Mexiquense, A.C., México (2003); es autor de un gran número de artículos científicos publicados en revistas indizadas en América Latina y Europa. Ha realizado estancias de investigación y docencia en Argentina, España, Polonia y Uruguay.

Beatriz Pico-González. Doctora en planeación estratégica y dirección de tecnología por la Universidad Popular Autónoma del estado de Puebla (UPAEP). Es egresada de la licenciatura en administración industrial por el Instituto Politécnico Nacional con especialidad en finanzas. Maestra en administración. Ha trabajado como especialista en proyectos de Ges- 
tión de la Calidad en la implementación de sistemas de calidad; asimismo como consultora en las Mi Pymes en temas de Planeación estratégica y calidad. Es coordinadora del doctorado en planeación estratégica y dirección de tecnología de la UPAEP, reconocido por el PNP y el Conacyt. Coordina un proyecto de "Emprendimiento en red para empresas de base tecnológica de nivel intermedio" con fondos de Conacyt. Es asesora en la coordinación con miembros de la Universidad Politécnica de Cataluña en España con el Proyecto "Diseño de acciones estratégicas para la sustentabilidad de las empresas familiares de giro educativo de nivel superior" y es miembro del CTNN9 del IMNC como revisora de las normas internacionales ISO 9000. Sus líneas de investigación son: estrategias e innovación tecnológica.

Francisco Javier Méndez-Ramírez. Es doctor en planeación estratégica y dirección tecnológica por la Universidad Popular Autónoma del estado de Puebla. Actualmente es profesor-investigador y consultor de empresas del Instituto Tecnológico y de Estudios Superiores de Monterrey, campus Puebla. Profesor-investigador de medio tiempo en la Benemérita Universidad Autónoma de Puebla en la Facultad de Ingeniería del Colegio de Ingeniería Industrial. Se ha desempeñado en el ámbito profesional como director de operaciones en empresas familiares del ramo industrial del plástico y alimentos. Es consultor en materia de emprendimiento y empresa familiar en el itesm-Puebla. Así también se desempeña como catedrático del área de negocios. Sus líneas de investigación son: impacto de la empresa familiar en el desarrollo económico de Puebla e impacto de liderazgo de género en la empresa familiar. 